\title{
PROBLÈMES D'EFFECTIVITÉ SUR LES QUARTIQUES DE FERMAT
}

\author{
Élie Cali et Alain Kraus
}

Let $K$ be a number field. An element $b \in K^{*}$ being given, let $C_{b}$ be the curve defined over $K$ by the equation $x^{4}+y^{4}=b z^{4}$. Let $C_{b}(K)$ be the set of the $K$-rational points of $C_{b}$. This paper uses Dem'janenko and Manin type methods to obtain effective criteria for $C_{b}(K)$ to be empty.

\section{INTRODUCTION}

Soit $K$ un corps de nombres. Étant donné un élément $b$ de $K^{*}$, on note $C_{b}$ la courbe définie sur $K$ d'équation

$$
x^{4}+y^{4}=b z^{4} \text {. }
$$

C'est une courbe lisse de genre 3. D'après les travaux de G. Faltings, l'ensemble $C_{b}(K)$ des points de $C_{b}$ rationnels sur $K$ est fini. On s'intéresse dans ce travail à l'effectivité de certaines méthodes globales concernant l'étude de $C_{b}(K)$. Soit $E_{b}$ la courbe elliptique définie sur $K$ d'équation

$$
Y^{2} Z=X^{3}-b X Z^{2}
$$

Les classes de $K$-isomorphisme de $C_{b}$ et $E_{b}$ ne dépendent que de la classe de $b$ modulo $K^{* 4}$. Il existe deux morphismes indépendants définis sur $K$ de $C_{b}$ sur $E_{b}$. En particulier, si $E_{b}(K)$ est de rang 0 , il est facile de déterminer $C_{b}(K)$. Dans le cas où $E_{b}(K)$ est de rang 1 , on obtient dans ce qui suit des critères effectifs entraînant que $C_{b}(K)$ est vide. Comme conséquence d'une étude des points rationnels de tordues galoisiennes de courbes sur les corps de nombres, J. Silverman a démontré en 1986 le résultat suivant ([8]).

THÉORÈmE. (Silverman) Il existe une constante absolue $c_{0}$, dépendant seulement du degré de $K$ sur $\mathbb{Q}$, telle que l'assertion suivante soit satisfaite.

Soient $b$ un élément de $K^{*}$ qui ne soit pas dans $K^{* 4}$ et $L$ une extension de $K$ obtenue en adjoignant à $K$ une racine quatrième de $b$. Alors, si $E_{b}(K)$ est de rang 1 et si la norme de $K$ sur $\mathbb{Q}$ du discriminant relatif de l'extension $L / K$ est plus grande que $c_{0}$, l'ensemble $C_{b}(K)$ est vide.

En particulier, pour toute classe d'élément $b$ dans $K^{*} / K^{* 4}$ sauf un nombre fini, si le rang de $E_{b}(K)$ est 1 , alors $C_{b}(K)$ est vide.

Received 31st July, 2006

Copyright Clearance Centre, Inc. Serial-fee code: 0004-9727/07 \$A2.00+0.00. 
Sa démonstration repose sur la mise en œuvre d'une méthode de Dem'janenko et Manin faisant intervenir des arguments de hauteurs. Silverman a démontré l'existence d'une constante $c_{0}$ dépendant exponentiellement du degré de $K$ sur $\mathbb{Q}$. Dans la première partie de ce travail, on se préoccupe de l'effectivité de cet énoncé en explicitant une telle constante $c_{0}$. On est confronté pour cela au problème de rendre effectif le théorème des zéros de Hilbert dans un cas particulier (appendice 1). Comme conséquence du résultat que l'on obtient à ce sujet, en notant $n$ le degré de $K$ sur $\mathbb{Q}$, on constate que l'on peut prendre

$$
c_{0}=\exp (48+6 n) \text {. }
$$

Cette constante s'avère inefficace d'un point de vue pratique. Dans le cas particulier où $K=\mathbb{Q}$, on dispose du résultat optimal suivant prouvé par Dem'janenko en 1968 ([2]).

ThÉorème. (Dem'janenko) Soit $b$ un entier $\geqslant 3$ sans puissances quatrièmes. Si $E_{b}(\mathbb{Q})$ est de rang $\leqslant 1$, alors $C_{b}(\mathbb{Q})$ est vide.

On généralise dans la deuxième partie cet énoncé aux corps totalement réels, avec une constante effective qui reste utilisable en pratique dans certaines situations. On s'est inspiré pour cela d'une méthode de G. Grigorov et J. Rizov qui leur a permis d'obtenir, si $K=\mathbb{Q}$, des estimations uniformes pour la différence entre la hauteur de Weil et la hauteur de Néron-Tate sur $E_{b}$, et d'en déduire une nouvelle démonstration du Théorème de Dem'janenko ([3]). On obtient le résultat suivant: le corps $K$ étant totalement réel, de degré $n$ sur $\mathbb{Q}$, soit $\left(u_{1}, \ldots, u_{n-1}\right)$ un système d'unités fondamentales de l'anneau d'entiers $O_{K}$ de $K$. Pour tout $x \in O_{K}$, notons $H(x)$ la hauteur de $x$ relative à $K$. On a

$$
H(x)=\prod_{\sigma} \operatorname{Max}(1,|\sigma(x)|)
$$

où $\sigma$ parcourt les $n$ plongements de $K$ dans $\mathbb{R}$. Notons par ailleurs $\mathrm{N}_{K / \mathbb{Q}}: K \rightarrow \mathbb{Q}$ l'application norme de $K$ sur $\mathbb{Q}$ et $v_{\mathfrak{p}}: K^{*} \rightarrow \mathbb{Z}$ la valuation sur $K$ standard normalisée associée à un idéal premier $\mathfrak{P}$ de $O_{K}$.

ThÉoRÈme. Soit $b$ un élément non nul de $O_{K}$. Supposons que les conditions suivantes soient satisfaites.

(1) pour tout idéal premier $\mathfrak{P}$ de $O_{K}$, on a $v_{\mathfrak{p}}(b)<4$.

(2) Le groupe $E_{b}(K)$ est de rang $\leqslant 1$.

(3) On a les inégalités

$$
\mathrm{N}_{K / \mathrm{Q}}(b)>2^{(11 n) / 2} \quad \text { et } \quad \mathrm{N}_{K / \mathrm{Q}}(b) \geqslant\left(\prod_{i=1}^{n-1} H\left(u_{i}\right)\right)^{4}
$$

Alors, l'ensemble $C_{b}(K)$ est vide.

Pour exploiter numériquement cet énoncé, il importe évidemment de savoir démontrer que, $b$ étant donné, le rang de $E_{b}(K)$ est au plus 1 , si tel est le cas. On peut effectuer pour 
cela une 2-descente comme il est expliqué dans [7]. Signalons à ce propos qu'il existe un programme, écrit par D. Simon, qui permet parfois de déterminer le rang d'une courbe elliptique sur un corps de nombres de degré sur $\mathbb{Q}$ assez petit ([9]).

Nous remercions vivement $\mathrm{E}$. Halberstadt qui nous a communiqué le résultat qui lui est dû figurant dans l'appendice 3. Nous remercions également le referee de cet article qui nous a indiqué que les résultats obtenus dans [5], ou bien dans [1], permettaient d'améliorer les énoncés du Théorème 1 et de son corollaire. Signalons que la constante $c_{0}$ que nous obtenions préalablement était $\exp (169+6 n)$.

\section{EFfectivité DU ThÉorème de Silverman}

Soient $\overline{\mathbb{Q}}$ une clôture algébrique de $\mathbb{Q}$ et $K$ un corps de nombres contenu dans $\overline{\mathbb{Q}}$. Considérons un élément non nul $b$ de $K$ qui n'est pas dans $K^{4}$ et choisissons une racine quatrième $\alpha$ de $b$ dans $\overline{\mathbb{Q}}$. Notons:

(a) $L$ le corps $K(\alpha)$.

(b) $d$ le degré de $L$ sur $K$; on a $d=4$ si et seulement si $b$ n'est pas un carré dans $K$ et $b$ n'est pas dans $-4 K^{4}$. On a $d=2$ sinon.

(c) $D_{L / K}$ le discriminant relatif de l'extension $L / K$; c'est un idéal de l'anneau d'entiers de $K$.

(d) $\mathrm{N}_{K / \mathbf{Q}}\left(D_{L / K}\right)$ la norme de $K$ sur $\mathbb{Q}$ de $D_{L / K}$.

(e) $\delta_{K}$ le nombre de places archimédiennes de $K$. Si $r_{1}$ (respectivement $2 r_{2}$ ) est le nombre de plongements réels (respectivement complexes) de $K$, on a $\delta_{K}=r_{1}+r_{2}$.

L'énoncé que l'on obtient est le suivant.

THÉORÈme 1. Supposons que le rang de $E_{b}(K)$ soit 1 . Alors, si l'on a

$$
\log N_{K / \mathbf{Q}}\left(D_{L / K}\right) \geqslant\left(3,98(d-1)+\delta_{K} \log d\right) d,
$$

l'ensemble $C_{b}(K)$ est vide.

En notant $D_{L}$ le discriminant de $L$ et $D_{K}$ celui de $K$, on a

$$
\left|D_{L}\right|=\mathrm{N}_{K / \mathbf{Q}}\left(D_{L / K}\right)\left|D_{K}\right|^{d},
$$

de sorte que l'inégalité (1) peut aussi s'écrire

$$
\log \left|D_{L}\right| \geqslant\left(3,98(d-1)+\delta_{K} \log d+\log \left|D_{K}\right|\right) d .
$$

Puisque le degré $n$ de $K$ sur $\mathbb{Q}$ vaut $r_{1}+2 r_{2}$, on déduit directement du théorème l'énoncé suivant signalé dans l'introduction.

COROlla IRE 1. Supposons que le rang de $E_{b}(K)$ soit 1 . Alors, si l'on a

$$
\mathrm{N}_{K / \mathrm{Q}}\left(D_{L / K}\right) \geqslant \exp (48+6 n)
$$

l'ensemble $C_{b}(K)$ est vide. 


\section{DÉMONSTRATION DU THÉORÈME 1}

1.1. Notations. Étant donné un corps de nombres $k$, on introduit les notations suivantes:

(a) $O_{k}$ son anneau d'entiers.

(b) $v_{\mathfrak{p}}: k^{*} \rightarrow \mathbb{Z}$ la valuation normalisée standard associée à un idéal premier $\mathfrak{P}$ de $O_{k}$.

(c) $M_{k}$ l'ensemble des places de $k$, c'est l'ensemble des classes d'équivalence de valeurs absolues usuelles sur $k$.

(d) $M_{k}^{\infty}$ l'ensemble des places archimédiennes de $k$. Si $v \in M_{k}^{\infty}$ correspond à un plongement $\sigma: k \rightarrow \mathbb{C}$, la valeur absolue normalisée associée à $v$ est définie pour tout $x \in k$ par la formule

$$
|x|_{v}=|\sigma(x)| .
$$

(e) $\quad M_{k}^{0}$ l'ensemble des places non archimédiennes de $k$. Soit $v$ une telle place correspondant à un idéal premier $\mathfrak{P}$ de $O_{k}$ de caractéristique résiduelle $p$. La place $v$ est représentée par la valeur absolue qui est définie pour tout $x \in k^{*}$ par la formule

$$
|x|_{v}=p^{-v_{\mathfrak{P}}(x) / e_{\mathfrak{P}}}
$$

où $e_{\mathfrak{P}}=v_{\mathfrak{p}}(p)$ est l'indice de ramification de $\mathfrak{P}$ sur $p$.

(f) Pour tout $v \in M_{k}$, on note $n_{v}$ le degré local de $v$. Si $v \in M_{k}^{\infty}$, on a $n_{v}=1$ ou $n_{v}=2$. On a $n_{v}=1$ si et seulement si $v$ correspond à un plongement de $k$ dans $\mathbb{R}$. Si $v \in M_{k}^{0}$ est associée à un idéal premier $\mathfrak{P}$ de $O_{k}$ au-dessus d'un nombre premier $p$, on a $n_{v}=e_{\mathfrak{p}} f_{\mathfrak{p}}$ où $f_{\mathfrak{p}}$ est le degré de $O_{k} / \mathfrak{P}$ sur $\mathbb{F}_{\mathfrak{p}}$.

1.2. Les hauteurs $h_{C}, h_{E}$ ET $\widehat{h}_{E}$. Rappelons la définition de l'application hauteur absolue logarithmique $h: \mathbb{P}^{2}(\overline{\mathbb{Q}}) \rightarrow \mathbb{R}$ définie sur l'ensemble des points du plan projectif $\mathbb{P}^{2}$ à valeurs dans $\overline{\mathbb{Q}}$. Soient $P=[x, y, z]$ un point de $\mathbb{P}^{2}(\overline{\mathbb{Q}})$ et $k$ un corps de nombres contenant $x, y$ et $z$. La hauteur absolue logarithmique $h(P)$ de $P$ est définie par la formule ([7, p. 215]).

$$
h(P)=\frac{1}{[k: \mathbb{Q}]} \sum_{v \in M_{k}} n_{v} \log \operatorname{Max}\left(|x|_{v},|y|_{v},|z|_{v}\right),
$$

où $[k: \mathbb{Q}]$ est le degré de $k$ sur $\mathbb{Q}$. La définition de $h(P)$ ne dépend pas du choix des coordonnées de $P$ choisies ni du corps de nombres $k$ les contenant.

Afin de simplifier les notations, on désigne dans la suite par $C$ et $E$ les courbes sur Q d'équations

$$
C: x^{4}+y^{4}=z^{4} \quad \text { et } \quad E: Y^{2} Z=X^{3}-X Z^{2} \text {. }
$$

On notera: 
(a) $h_{C}$ la restriction de $h$ à $C(\bar{Q})$.

(b) $h_{E}$ la hauteur sur $E$ relative à la fonction $X / Z$ (loco citato). En posant $O=[0,1,0]$, on a $h_{E}(O)=0$. Pour tout point $M=[X, Y, Z] \in E(\overline{\mathbb{Q}})$ distinct de $O$ et tout corps $k$ contenant $X$ et $Z$, on a l'égalité:

$$
h_{E}(M)=\frac{1}{[k: \mathbb{Q}]} \sum_{v \in M_{k}} n_{v} \log \operatorname{Max}\left(|X|_{v},|Z|_{v}\right) .
$$

Cette définition ne dépend pas du choix des coordonnées de $M$.

(c) $\widehat{h}_{E}: E(\overline{\mathbb{Q}}) \rightarrow \mathbb{R}$ la hauteur de Néron-Tate sur $E([\mathbf{7}, \mathrm{p} .228])$. On a $2 \widehat{h}_{E}=h_{E}+O(1)$ (voir loco citato, Theorem 9.3, p. 229). Comme nous l'a signalé le referee de cet article, les résultats qui se trouvent dans [5] ou [1] ont été implantés sur ordinateur par les auteurs. Leur programme permet d'obtenir, pour tout point $M \in E(\overline{\mathbb{Q}})$, l'inégalité:

$$
\left|\widehat{h}_{E}(M)-\frac{1}{2} h_{E}(M)\right| \leqslant 0,232 .
$$

1.3. Une majoration De hauteur. On dispose de deux morphismes $\phi$ et $\psi$ sur $\mathbb{Q}$ de $C$ sur $E$ définis pour tout point $[x, y, z]$ de $C$ par les formules

$$
\phi([x, y, z])=\left[-x^{2} z, x y^{2}, z^{3}\right] \text { et } \psi([x, y, z])=\left[-y^{2} z, x^{2} y, z^{3}\right] .
$$

On va démontrer le résultat suivant.

THÉORÈme 2. Soit $P$ un point de $C(\overline{\mathbb{Q}})$. Supposons que $\phi(P)$ et $\psi(P)$ dans $E(\overline{\mathbb{Q}})$ soient linéairement dépendants sur $\mathbf{Z}$. Alors, on a

$$
h_{C}(P)<1,99 .
$$

DÉmonstration: Indiquons d'abord le principe de la démonstration. Soit $\phi+\psi$ : $C \rightarrow E$ le morphisme somme de $\phi$ et $\psi$ relatif à loi de groupe sur $E$. On fournit des majorations explicites des quantités

et

$$
\begin{aligned}
& \left|h_{C}(P)-\widehat{h}_{E}(\phi(P))\right|, \\
& \left|h_{C}(P)-\widehat{h}_{E}(\psi(P))\right|, \\
& \left|2 h_{C}(P)-\widehat{h}_{E}((\phi+\psi)(P))\right| .
\end{aligned}
$$

Notons $\{\rangle:, E(\overline{\mathbb{Q}}) \times E(\overline{\mathbb{Q}}) \rightarrow \mathbb{R}$ l'accouplement de Néron-Tate sur $E$ (voir [7, p. 232]). On a

$$
\begin{aligned}
\langle\phi(P), \phi(P)\rangle & =2 \widehat{h}_{E}(\phi(P)) \\
\langle\psi(P), \psi(P)\rangle & =2 \widehat{h}_{E}(\psi(P)) \\
\langle\phi(P), \psi(P)\rangle & =\widehat{h}_{E}((\phi+\psi)(P))-\widehat{h}_{E}(\phi(P))-\widehat{h}_{E}(\psi(P))
\end{aligned}
$$


Les points $\phi(P)$ et $\psi(P)$ étant linéairement dépendants dans $E(\overline{\mathbb{Q}})$, le déterminant de la matrice

$$
\left(\begin{array}{cc}
\langle\phi(P), \phi(P)\rangle & \langle\phi(P), \psi(P)\rangle \\
\langle\phi(P), \psi(P)\rangle & \langle\psi(P), \psi(P)\rangle
\end{array}\right)
$$

est nul. Les majorations obtenues ci-dessus permettent alors d'obtenir le résultat annoncé.

Posons $P=[x, y, z] \in C(\overline{\mathbb{Q}})$. Pour toute la suite de la démonstration, on considère un corps de nombres $k$ contenant $x, y$ et $z$. On supposera, ce qui n'est pas restrictif, que $x, y$ et $z$ sont dans $O_{k}$.

\section{Proposition 1. On a l'inégalité}

$$
\left|4 h_{C}(P)-h_{E}((\phi+\psi)(P))\right| \leqslant \log 69 .
$$

DÉMONSTRATION: On vérifie que l'on a

$$
(\phi+\psi)([x, y, z])=[U, V, W],
$$

avec

$$
\begin{gathered}
U=(x+y) z\left(x^{2}+x y+y^{2}\right)^{2}, \quad V=-x y\left(x^{2}+x y+y^{2}\right)\left(2 x^{2}+3 y x+2 y^{2}\right), \\
W=(x+y)^{3} z^{3} .
\end{gathered}
$$

Vérifions l'inégalité $(7)$ si $(x+y) z=0$. On a dans ce cas $U=W=0$ et $(\phi+\psi)(P)=O$, d'où $h_{E}((\phi+\psi)(P))=0$.

Si $z=0$, on a $x y \neq 0$ et pour tout $v \in M_{k}$ on a $|x|_{v}=|y|_{v}$, ce qui conduit d'après la formule du produit à $h_{C}(P)=0$ (formule (2)).

Supposons $x+y=0$. On a alors $2 x^{4}=z^{4}, x y z \neq 0$ et $P=[1,-1, \beta]$, où $\beta \in \overline{\mathbb{Q}}$ vérifie l'égalité $\beta^{4}=2$. Prenons pour $k$ le corps $\mathbb{Q}(\beta)$. On a alors $r_{1}=2, r_{2}=1$. Pour toute place $v \in M_{k}^{\infty}$, on a $|\beta|_{v}=2^{1 / 4}$ où $2^{1 / 4}$ est la racine quatrième positive de 2 dans $\mathbb{R}$. Par ailleurs, $\beta$ étant dans $O_{k}$, on a $|\beta|_{v} \leqslant 1$ pour toute place finie $v \in M_{k}^{0}$. Il en résulte que l'on a

$$
h_{C}(P)=\frac{1}{4}\left(4 \log 2^{1 / 4}\right)=\frac{1}{4} \log 2 .
$$

Puisque $4 h_{C}(P)=\log 2$ est plus petit que $\log 69$, l'inégalité (7) est donc vraie si $x+y=0$. D'où notre assertion.

Supposons désormais $(x+y) z$ non nul. Dans ce cas, on a (formule (3))

$$
h_{E}((\phi+\psi)(P))=\frac{1}{[k: \mathbb{Q}]} \sum_{v \in M_{k}} n_{v} \log \operatorname{Max}\left(\left|x^{2}+x y+y^{2}\right|_{v}^{2},|(x+y) z|_{v}^{2}\right) .
$$

Pour tout $v \in M_{k}^{0}$ on a

et

$$
\begin{aligned}
\left|x^{2}+x y+y^{2}\right|_{v} & \leqslant \operatorname{Max}\left(|x|_{v},|y|_{v},|z|_{v}\right)^{2} \\
|(x+y) z|_{v} & \leqslant \operatorname{Max}\left(|x|_{v},|y|_{v},|z|_{v}\right)^{2} .
\end{aligned}
$$


Par ailleurs, pour tout $v \in M_{k}^{\infty}$ on a

et

$$
\begin{aligned}
\left|x^{2}+x y+y^{2}\right|_{v} & \leqslant 3 \operatorname{Max}\left(|x|_{v},|y|_{v},|z|_{v}\right)^{2} \\
|(x+y) z|_{v} & \leqslant 2 \operatorname{Max}\left(|x|_{v},|y|_{v},|z|_{v}\right)^{2} .
\end{aligned}
$$

On en déduit l'inégalité

$$
\begin{aligned}
h_{E}((\phi+\psi)(P)) \leqslant \frac{1}{[k: \mathbb{Q}]} \sum_{v \in M_{k}^{0}} n_{v} & \log \operatorname{Max}\left(|x|_{v},|y|_{v},|z|_{v}\right)^{4} \\
& +\frac{1}{[k: \mathbb{Q}]} \sum_{v \in M_{k}^{\infty}} n_{v} \log \left(9 \operatorname{Max}\left(|x|_{v},|y|_{v},|z|_{v}\right)^{4}\right) .
\end{aligned}
$$

Compte tenu du fait que

$$
[k: \mathbb{Q}]=\sum_{v \in M_{k}^{\infty}} n_{v}
$$

il en résulte que l'on a

$$
h_{E}((\phi+\psi)(P)) \leqslant \log 9+4 h_{C}(P) .
$$

Inversement, démontrons que l'on a

$$
4 h_{C}(P) \leqslant \log 69+h_{E}((\phi+\psi)(P)) .
$$

On utilise pour cela la proposition de l'appendice 1. Posons

$$
g=\left(x^{2}+x y+y^{2}\right)^{2} \text { et } h=(x+y)^{2} z^{2} .
$$

Le point $[x, y, z]$ appartenant à $C(\overline{\mathbb{Q}})$ on a $x^{4}+y^{4}=z^{4}$. Par suite, les égalités (1) et (2) de cette proposition entraînent, avec ses notations,

$$
\begin{aligned}
& x^{12}=Q(x, y, z) g+R(x, y, z) h, \\
& y^{12}=Q(y, x, z) g+R(y, x, z) h, \\
& z^{12}=2 z^{8} g-z^{6}(x+y)^{2} h .
\end{aligned}
$$

Pour toute place finie $v \in M_{k}^{0}$, les éléments $x, y$ et $z$ étant dans $O_{k}$, les valeurs absolues $v$-adiques des éléments $Q(x, y, z), R(x, y, z), Q(y, x, z)$ et $R(y, x, z)$ sont inférieures à $\operatorname{Max}\left(|x|_{v},|y|_{v},|z|_{v}\right)^{8}$. On en déduit que

$$
\operatorname{Max}\left(|x|_{v},|y|_{v},|z|_{v}\right)^{4} \leqslant \operatorname{Max}\left(|g|_{v},|h|_{v}\right) .
$$

Pour toute place $v \in M_{k}^{\infty}$ on obtient dans ce cas l'inégalité (voir les coefficients des polynômes $Q$ et $R$ de la proposition de l'appendice 1)

$$
\operatorname{Max}\left(|x|_{v},|y|_{v},|z|_{v}\right)^{4} \leqslant 69 \operatorname{Max}\left(|g|_{v},|h|_{v}\right) .
$$

L'égalité (8) entraine alors l'inégalité (10). La proposition se déduit alors des conditions (9) et (10). 
Proposition 2. On a les inégalités

$$
\left|2 h_{C}(P)-h_{E}(\phi(P))\right| \leqslant \frac{\log 2}{2} \text { et }\left|2 h_{C}(P)-h_{E}(\psi(P))\right| \leqslant \frac{\log 2}{2} .
$$

DÉmOnStration: Par symétrie par rapport à $x$ et $y$, il suffit de démontrer la première inégalité de (11). Supposons $z=0$. On a $\phi(P)=[0,1,0]$ et $h_{E}(\phi(P))=0$. Par ailleurs, on a $h_{C}(P)=0$ comme on l'a déjà constaté dans la démonstration de la proposition 1. D'où le résultat dans ce cas. Supposons $z$ non nul. D'après les formules (5), on a alors

$$
h_{E}(\phi(P))=\frac{1}{[k: \mathbb{Q}]} \sum_{v \in M_{k}} n_{v} \log \operatorname{Max}\left(|x|_{v}^{2},|z|_{v}^{2}\right) .
$$

Posons $a=x^{2}$ et $b=z^{2}$. On a $x^{4}+y^{4}=z^{4}$. On en déduit pour tout $v \in M_{k}^{0}$ l'inégalité $|y|_{v}^{2} \leqslant \operatorname{Max}\left(|a|_{v},|b|_{v}\right)$. Par suite, on a dans ce cas

$$
\operatorname{Max}\left(|x|_{v},|y|_{v},|z|_{v}\right)^{2} \leqslant \operatorname{Max}\left(|a|_{v},|b|_{v}\right) .
$$

Par ailleurs, pour tout $v \in M_{k}^{\infty}$ on a $|y|_{v}^{2} \leqslant \sqrt{2} \operatorname{Max}\left(|a|_{v},|b|_{v}\right)$ et l'on obtient ainsi.

$$
\operatorname{Max}\left(|x|_{v},|y|_{v},|z|_{v}\right)^{2} \leqslant \sqrt{2} \operatorname{Max}\left(|a|_{v},|b|_{v}\right) .
$$

Il en résulte l'inégalité

$$
2 h_{C}(P) \leqslant \frac{\log 2}{2}+h_{E}(\phi(P)) .
$$

Inversement, pour tout $v \in M_{k}$ on a

$$
|a|_{v},|b|_{v} \leqslant \operatorname{Max}\left(|x|_{v},|y|_{v},|z|_{v}\right)^{2}
$$

d'où $h_{E}(\phi(P)) \leqslant 2 h_{C}(P)$ et le résultat.

Corollaire 2. On a les inégalités

$$
\begin{aligned}
\left|h_{C}(P)-\widehat{h}_{E}(\phi(P))\right| & \leqslant 0,406, \\
\left|h_{C}(P)-\widehat{h}_{E}(\psi(P))\right| & \leqslant 0,406, \\
\left|2 h_{C}(P)-\widehat{h}_{E}((\phi+\psi)(P))\right| & \leqslant 2,35 .
\end{aligned}
$$

DÉmonstration: C'est une conséquence directe de l'inégalité (4) ainsi que des Propositions 1 et 2 .

Terminons maintenant la démonstration du Théorème 2. Posons pour cela

et

$$
\begin{aligned}
\widehat{h}_{E}(\phi(P)) & =h_{C}(P)+\delta \\
\widehat{h}_{E}(\psi(P)) & =h_{C}(P)+\beta \\
\widehat{h}_{E}((\phi+\psi)(P)) & =2 h_{C}(P)+\gamma
\end{aligned}
$$


En exprimant le fait que le déterminant de la matrice

$$
\left(\begin{array}{cc}
2\left(h_{C}(P)+\delta\right) & \gamma-(\delta+\beta) \\
\gamma-(\delta+\beta) & 2\left(h_{C}(P)+\beta\right)
\end{array}\right)
$$

est nul, on obtient l'égalité

$$
h_{C}(P)^{2}+(\delta+\beta) h_{C}(P)+\delta \beta-\frac{(\gamma-(\delta+\beta))^{2}}{4}=0 .
$$

Le discriminant de cette équation est $2 \delta^{2}+2 \beta^{2}+\gamma^{2}-2 \gamma(\delta+\beta)$. Il est donc majoré en valeur absolue par

$$
2 \delta^{2}+2 \beta^{2}+\gamma^{2}+2|\gamma||\delta|+2|\gamma||\beta|
$$

donc, d'après le Corollaire 2 , par 10 . Il en résulte que l'on a

$$
h_{C}(P)<\frac{|\delta|+|\beta|+\sqrt{10}}{2}<1,99
$$

d'où le théorème.

1.4. Fin DE LA DÉmonstration DU ThÉORÈme 1. Rappelons que $\alpha$ désigne une racine quatrième de $b$ dans $\bar{Q}$. Les courbes $C_{b}$ et $C$ sont isomorphes sur $L=K(\alpha)$ via le morphisme $f_{b}: C_{b} \rightarrow C$ défini pour tout point $[x, y, z]$ de $C_{b}$ par l'égalité

$$
f_{b}([x, y, z])=[x, y, \alpha z] .
$$

Le corps $L$ est un corps minimal, au sens de l'appendice 2, sur lequel les courbes $C_{b}$ et $C$ sont isomorphes. En effet, par hypothèse $b$ n'est pas une puissance quatrième dans $K$, donc $C$ et $C_{b}$ ne sont pas isomorphes sur $K$ (on peut le vérifier en utilisant le Théorème 2.2 de $\left[7\right.$, p. 285]). L'assertion est donc immédiate si $d=2$. Supposons $d=4$ et $C_{b}$ isomorphe à $C$ sur une extension quadratique $H$ de $K$ contenue dans $L$. Dans ce cas, $b$ est alors une puissance quatrième dans $H$, ce qui conduit à une contradiction, d'où l'assertion.

Supposons que $C_{b}(K)$ ne soit pas vide. Considérons un point $P \in C_{b}(K)$. Posons $P=[x, y, z]$ (où $x, y$ et $z$ sont dans $O_{K}$ ) et $Q=f_{b}(P) \in C(L)$. On est dans l'un des deux cas intervenant dans l'énoncé du théorème de l'appendice 2.

Supposons que l'on soit dans le premier cas de ce théorème, c'est a dire qu'il existe $\sigma$ dans le groupe de Galois de $\bar{Q}$ sur $K$ tel que ${ }^{\sigma} f_{b} \circ f_{b}^{-1}$ ne soit pas l'identité de $C$ et fixe le point $Q$. On a l'égalité

$$
{ }^{\sigma} f_{b} \circ f_{b}^{-1}(Q)=[x, y, \sigma(\alpha) z],
$$

ce qui conduit à $z(\sigma(\alpha)-\alpha)=0$. On a $\sigma(\alpha) \neq \alpha$ car ${ }^{\sigma} f_{b}$ est distinct de $f_{b}$. Il en résulte que $z=0$. On en déduit que $x y \neq 0$ puis que $P=[x / y, 1,0]$ où $x / y$ est une 
racine primitive huitième de l'unité. En particulier, le groupe $\mu_{4}$ des racines quatrièmes de l'unité est contenu dans $K$. Soient $i$ un générateur de $\mu_{4}$ et $[i]$ l'automorphisme de $E_{b}$ défini par

$$
[i](X, Y, Z)=[-X, i Y, Z] \text {. }
$$

Le groupe $E_{b}(K)$ est alors muni de la structure de $\mathbb{Z}[i]$-module définie pour tous $a, b \in \mathbb{Z}$ et $M \in E_{b}(K)$ par l'égalité

$$
(a+i b) \cdot M=a M+b[i](M) .
$$

Ainsi, $E_{b}(K)$ modulo son sous-groupe de torsion est un $\mathbb{Z}[i]$-module libre de rang $r / 2$ où $r$ est le rang usuel de $E_{b}(K)$. L'entier $r$ est donc pair ce qui contredit le fait que $r=1$.

La condition 2 du théorème de l'appendice 2 est donc satisfaite, autrement dit on a

$$
\log \mathrm{N}_{K / \mathbf{Q}} D_{L / K} \leqslant\left(2(d-1) h_{C}(Q)+\delta_{K} \log d\right) d .
$$

Les morphismes $\phi$ et $\psi$ étant définis par les formules (5), vérifions que les points

$$
\phi(Q) \text { et } \psi(Q) \in E(L),
$$

sont $\mathbb{Z}$-linérairement dépendants dans $E(L)$. Les courbes elliptiques $E_{b}$ et $E$ sont isomorphes sur $L$ via le morphisme $g_{b}: E_{b} \rightarrow E$ défini pour tout point $[X, Y, Z]$ de $E_{b}$ par l'égalité

$$
g_{b}([X, Y, Z])=\left[\frac{X}{\alpha^{2}}, \frac{Y}{\alpha^{3}}, Z\right] .
$$

Par ailleurs, on dispose de deux morphismes $\phi_{b}: C_{b} \rightarrow E_{b}$ et $\psi_{b}: C_{b} \rightarrow E_{b}$ définis sur $K$ par les égalités

et

$$
\begin{aligned}
& \phi_{b}([x, y, z])=\left[-x^{2} z, x y^{2}, z^{3}\right] \\
& \psi_{b}([x, y, z])=\left[-y^{2} z, y x^{2}, z^{3}\right] .
\end{aligned}
$$

On vérifie directement que l'on a

$$
g_{b}^{-1} \circ \phi \circ f_{b}=\phi_{b} \quad \text { et } \quad g_{b}^{-1} \circ \psi \circ f_{b}=\psi_{b} .
$$

Il en résulte que les points

$$
g_{b}^{-1} \circ \phi \circ f_{b}(P) \text { et } g_{b}^{-1} \circ \psi \circ f_{b}(P),
$$

appartiennent à $E_{b}(K)$. Puisque le rang de $E_{b}(K)$ est 1 , ces points sont donc Z-dépendants sur $E_{b}(K)$. Le fait que $g_{b}$ soit un isomorphisme défini sur $L$ de $E_{b}$ sur $E$ entraîne alors notre assertion. D'après le Théorème 2 , on a donc l'inégalité

$$
h_{C}(Q)<1,99 \text {. }
$$

On déduit alors de (12) que l'on a

$$
\log \mathrm{N}_{K / \mathrm{Q}} D_{L / K}<\left(3,98(d-1)+\delta_{K} \log d\right) d,
$$

ce qui contredit l'inégalité (1) du Théorème 1. Cela termine sa démonstration. 


\section{ThÉoRÈme DE DEM'JANENKo ET CORPS TOTALEMENT RÉElS}

On considère dans cette deuxième partie un corps de nombres $K$ totalement réel, de degré $n$ sur $\mathbb{Q}$, d'anneau d'entiers $O_{K}$. Pour tout $x$ de $O_{K}$, notons $H(x)$ la hauteur de $x$ relative à $K$. On a

$$
H(x)=\prod_{\sigma} \operatorname{Max}(1,|\sigma(x)|),
$$

où $\sigma$ parcourt les $n$ plongements de $K$ dans $\mathbb{R}$. Le groupe des unités de $O_{K}$ modulo $\{ \pm 1\}$ est un $\mathbb{Z}$-module libre de rang $n-1$. Soit $\left(u_{1}, \ldots, u_{n-1}\right)$ un système d'unités fondamentales de $O_{K}$. On a l'énoncé suivant signalé dans l'introduction.

THÉORÈme 3. Soit $b$ un élément non nul de $O_{K}$. Supposons que les conditions suivantes soient satisfaites.

(1) pour tout idéal premier $\mathfrak{P}$ de $O_{K}$, on a $v_{\mathfrak{p}}(b)<4$.

(2) Le groupe $E_{b}(K)$ est de rang $\leqslant 1$.

(3) On a les inégalités

$$
\mathrm{N}_{K / \mathrm{Q}}(b)>2^{(11 n) / 2} \text { et } \quad \mathrm{N}_{K / \mathrm{Q}}(b) \geqslant\left(\prod_{i=1}^{n-1} H\left(u_{i}\right)\right)^{4} .
$$

Alors, l'ensemble $C_{b}(K)$ est vide.

Comme conséquence de la démonstration de ce théorème, les conditions 1 et 2 étant toujours supposées réalisées, si de plus pour tout plongement $\sigma$ de $K$ dans $\mathbb{R}$ on a $\sigma(b) \geqslant 1$, on obtient l'implication

$$
\mathrm{N}_{K / \mathrm{Q}}(b)>2^{(11 n) / 2} \Longrightarrow C_{b}(K)=\emptyset
$$

Par ailleurs, il convient de signaler que si $O_{K}$ est principal, il n'y a qu'un nombre fini d'éléments de $K^{*} / K^{* 4}$ pour lesquels il n'existe pas de représentants satisfaisant les conditions 1 et 3 du théorème. Il n'en va pas de même si $O_{K}$ n'est pas principal. Par exemple, si le nombre de classes de $K$ est 3 , on peut démontrer qu'il existe une infinité d'éléments de $K^{*} / K^{* 4}$ pour lesquels il n'existe pas de représentants $b \in O_{K}$ satisfaisant la condition 1. Le Théorème 3 est donc un cas particulier effectif du Théorème de Silverman seulement dans le cas où $O_{K}$ est principal.

DÉmONSTRATION DU ThÉORÈme 3. On reprend les notations du paragraphe 1.1, à ceci près que pour toute place finie $v \in M_{K}^{0}$, on note ici $v: K^{*} \rightarrow \mathbb{Z}$ la valuation standard normalisée associée à l'idéal premier de $O_{K}$ qui lui correspond. Avec cette notation, si $v$ est de caractéristique résiduelle $p$, on a pour tout $x \in K^{*}$

$$
|x|_{v}=p^{-v(x) / e_{v}},
$$


où $e_{v}$ est l'indice de ramification de $v$ sur $p$.

Soit $b$ un élément de $O_{K}$ vérifiant les deux premières conditions du Théorème 3 . Il s'agit de montrer que si la norme de $K$ sur $\mathbb{Q}$ de $b$ est assez grande, comme il est précisé dans l'énoncé de ce théorème, l'ensemble $C_{b}(K)$ est vide.

2.1. RÉduction sur $b$. S'il existe un plongement $\sigma$ de $K$ dans $\mathbb{R}$ tel que $\sigma(b)<0$, il est immédiat que $C_{b}(K)$ est vide et le théorème est démontré dans ce cas. On peut donc supposer que $b$ est totalement positif, autrement dit que pour tout plongement $\sigma: K \rightarrow \mathbb{R}$, on a $\sigma(b)>0$. Compte tenu de l'appendice 3 et de la deuxième inégalité de la condition (13), il existe une unité $u$ de $O_{K}$ telle que pour tout plongement $\sigma$ de $K$ dans $\mathbb{R}$ on ait $\sigma\left(b u^{4}\right) \geqslant 1$. Par ailleurs, les courbes $C_{b}$ et $C_{b u^{4}}$ sont $K$-isomorphes et si l'on remplace $b$ par $b u^{4}$ les trois conditions intervenant dans l'énoncé du Théorème 3 ne changent pas. Quitte à remplacer $b$ par $b u^{4}$, on peut donc supposer, ce que l'on fera dans toute la suite, que la condition suivante est satisfaite:

$$
\text { pour tout plongement } \sigma: K \rightarrow \mathbb{R} \text {, on a } \sigma(b) \geqslant 1 .
$$

2.2. La courbe elliptique $E_{b}$. Afin de simplifier les notations, on notera dans la suite $E_{b}$ la courbe affine d'équation de Weierstrass

$$
y^{2}=x^{3}-b x
$$

Les invariants standard $c_{4}, c_{6}$ et $\Delta$ associés à cette équation sont (voir [10]):

$$
c_{4}=2^{4} \cdot 3 \cdot b, \quad c_{6}=0 \quad \text { et } \quad \Delta=2^{6} \cdot b^{3} .
$$

La courbe $E_{b}$ a bonne réduction en toutes les places finies $v \in M_{K}^{0}$ pour lesquelles on a $v(2 b)=0$. Son invariant modulaire est 1728 , il est entier, donc $E_{b}$ a partout potentiellement bonne réduction.

Lemme 1. Soit $v$ une place finie de $K$ telle que $v(b)>0$. Alors, le modèle (16) est minimal en $v$ et $E_{b}$ a mauvaise réduction de type additif en $v$.

DÉmonstration: Posons $m=v(b)$ et $e=v(2)$. D'après la condition $1 \mathrm{du}$ Théorème 3, on a $m=1,2$ ou 3. Cela entraîne l'assertion si $e=0$. Supposons $e \geqslant 1$. D'après (17), on a

$$
v\left(c_{4}\right)=4 e+m \quad \text { et } \quad v(\Delta)=6 e+3 m .
$$

Supposons que le modèle (16) ne soit pas minimal en $v$, autrement dit qu'il ne soit pas minimal sur le complété $K_{v}$ de $K$ en $v$. Dans ce cas, il existe un élément $u \in K_{v}$ de valuation $>0$ tel que

$$
\frac{c_{4}}{u^{4}}, \quad \frac{c_{6}}{u^{6}}(=0) \text { et } \frac{\Delta}{u^{12}},
$$


appartiennent à l'anneau de valuation de $K_{v}$ et soient les invariants standard associés à une courbe elliptique sur $K_{v}$. En notant encore $v$ le prolongement de $v$ à $K_{v}$, on déduit de (18) les inégalités

$$
0<v\left(\frac{c_{4}}{u^{4}}\right)<4 e \quad \text { et } \quad v\left(\frac{c_{4}}{u^{4}}\right) \not \equiv 0 \text { mod. } 4 .
$$

Cela contredit le Théorème 2 de [4] et la remarque qui le suit. D'où le résultat.

Considérons une place finie $v$ de $K$ telle que $v(b)>0$. Soient $K_{v}$ le complété de $K$ en $v$ et $k_{v}$ le corps résiduel. On déduit du Lemme 1 que la courbe sur $k_{v}$ déduite de la courbe elliptique $E_{b}$ par réduction possède un unique point singulier qui est $(0,0)$ (voir $\left[7\right.$, p. 173]). On désigne par $E_{b}^{0}\left(K_{v}\right)$ le sous-groupe de $E_{b}\left(K_{v}\right)$ formé des points de réduction non singulière sur $k_{v}$.

Lemme 2. Supposons $v(b)>0$. Soit $P=(x, y)$ un point de $E_{b}\left(K_{v}\right)$. Alors, $P$ appartient à $E_{b}^{0}\left(K_{v}\right)$ si et seulement si $v(x) \leqslant 0$.

DÉMONSTRATION: Le modèle (16) étant minimal sur $K_{v}$, la condition annoncée est clairement nécessaire, car si $v(x)>0$ on a aussi $v(y)>0$. Inversement, supposons $v(x) \leqslant 0$. Posons $x=\pi^{n} a$, où $\pi$ est une uniformisante de $K_{v}, n \leqslant 0$ et $v(a)=0$. Si $n=0$, on a $v(y) \geqslant 0$ et $P$ ne se réduit pas en $(0,0)$. Si $n<0$, on a $2 v(y)=3 v(x)$ et $P$ se réduit en le point $[0,1,0]$ sur la courbe projective réduite $Y^{2} Z=X^{3}$, en particulier $P \in E_{b}^{0}\left(K_{v}\right)$. D'où le lemme.

Pour tout point $P=(x, y) \in E_{b}(K)$ à distance finie, on notera désormais

et

$$
\begin{aligned}
& H_{x}(P)=\prod_{v \in M_{K}} \operatorname{Max}\left(1,|x|_{v}\right)^{n_{v} / n} \\
& h_{x}(P)=\log H_{x}(P)
\end{aligned}
$$

où, comme dans le paragraphe $1.1, n_{v}$ est le degré local en $v$. On pose $H_{x}(O)=1$, où $O$ est le point à l'infini de $E_{b}$.

2.3. Le sous-groupe $\Gamma$ DE $E_{b}(K)$. On va maintenant définir un sous-groupe $\Gamma$ d'indice fini de $E_{b}(K)$. Considérons pour cela le sous-ensemble $G$ de $E_{b}(K)$ formé des points qui appartiennent à $E_{b}^{0}\left(K_{v}\right)$ pour toute place finie $v \in M_{K}^{0}$ telle que $v(b)>0$. D'après le Lemme 2, étant donné $P=(x, y) \in E_{b}(K)$, on a l'équivalence

$$
P \in G \Longleftrightarrow v(x) \leqslant 0 \text { pour toute place } v \in M_{K}^{0} \text { telle que } v(b)>0 .
$$

C'est un sous-groupe d'indice fini de $E_{b}(K)$ car $E_{b}^{0}\left(K_{v}\right)$ est lui-même un sous-groupe d'indice fini de $E_{b}\left(K_{v}\right)\left(\left[7\right.\right.$, p. 359]). Pour tout plongement $\sigma: K \rightarrow \mathbb{R}$, notons $E_{\sigma(b)}$ la courbe elliptique définie sur $\mathbf{R}$ d'équation

$$
y^{2}=x^{3}-\sigma(b) x
$$


Puisque les trois abscisses des points d'ordre 2 de $E_{\sigma(b)}$ sont réelles, l'ensemble $E_{\sigma(b)}(\mathbb{R})$ possède deux composantes connexes. On désigne par $I_{\sigma}$ la composante connexe de l'élément neutre. Compte tenu du fait que l'on a $\sigma(b)>0$, pour tout point $M=(x, y)$ $\in E_{\sigma(b)}(\mathbb{R})$, on a l'équivalence (en prenant la racine carrée positive)

$$
M \in I_{\sigma} \Longleftrightarrow x \geqslant \sqrt{\sigma(b)} .
$$

Par ailleurs, pour tout $M \in E_{\sigma(b)}(\mathbb{R})$, le point $2 M$ appartient à $I_{\sigma}$. On définit alors $\Gamma$ comme étant le sous-ensemble de $G$ formé des points $P \in G$ tels que $\sigma(P)$ appartienne à $I_{\sigma}$ pour tout plongement $\sigma: K \rightarrow \mathbb{R}$. C'est un sous-groupe de $G$ et d'après l'assertion précédente, il est d'indice 2 dans $G$. En particulier, $\Gamma$ est un sous-groupe d'indice fini de $E_{b}(K)$.

2.4. Hauteurs sur $\Gamma$. Pour tout idéal $\mathscr{I}$ de $O_{K}$, on notera dans toute la suite $\mathrm{N}(\mathscr{I})$ la norme de $K$ sur $\mathbb{Q}$ de $\mathscr{I}$.

LEMME 3. Soit $Q=(x, y)$ un point de $E_{b}(K)$. Supposons que pour tout plongement $\sigma: K \rightarrow \mathbb{R}$, le point $\sigma(Q)$ appartienne à $I_{\sigma}$. On a l'égalité

$$
H_{x}(Q)^{n}=\prod_{\left\{p ; v_{p}(x)>0\right\}} \mathrm{N}(\mathfrak{p})^{v_{p}(x)}
$$

le produit étant indexé par l'ensemble des idéaux premiers p de $O_{K}$ tels que $v_{\mathfrak{p}}(x)>0$.

DÉmonstration: D'après (19), on a

$$
H_{x}(P)^{n}=\prod_{\nu \in M_{K}^{0}} \operatorname{Max}\left(1,|x|_{v}\right)^{n_{v}} \prod_{v \in M_{K}^{\infty}} \operatorname{Max}\left(1,|x|_{v}\right)^{n_{v}} .
$$

Soit $v$ une place finie. La formule (14) entraine l'égalité

$$
|x|_{v}^{n_{v}}=q_{v}^{-v(x)},
$$

où $q_{v}$ est le cardinal du corps résiduel de $v$. Si p est l'idéal premier de $O_{K}$ correspondant à $v$, on a donc

$$
|x|_{v}=\mathrm{N}(\mathfrak{p})^{-v_{p}(x)}
$$

Il en résulte que l'on a

$$
\prod_{v \in M_{K}^{0}} \operatorname{Max}\left(1,|x|_{v}\right)^{n_{v}}=\prod_{\left\{\mathfrak{p} ; v_{p}(x)<0\right\}} \mathrm{N}(\mathfrak{p})^{-v_{p}(x)} .
$$

Par ailleurs, l'hypothèse faite et les conditions (21) et (15) entrainent que pour tout plongement $\sigma: K \rightarrow \mathbb{R}$, on a $\sigma(x) \geqslant \sqrt{\sigma(b)} \geqslant 1$. Les degrés locaux $n_{v}$ étant égaux à 1 pour les places infinies, on obtient

$$
\prod_{v \in M_{K}^{\infty}} \operatorname{Max}\left(1,|x|_{v}\right)^{n_{v}}=\left|N_{K / Q}(x)\right|=\prod_{p} N(p)^{v_{D}(x)} .
$$


Les égalités (22) et (23) entraînent alors le lemme.

Considérons maintenant un point $P=(x, y) \in E_{b}(K)$ tel que $2 P \neq O$. L'abscisse de $2 P$ est donnée par l'égalité

$$
x(2 P)=\frac{\left(x^{2}+b\right)^{2}}{4 x\left(x^{2}-b\right)} .
$$

Pour tout idéal premier $\mathfrak{p}$ de $O_{K}$ posons $\alpha(\mathfrak{p})=v_{\mathfrak{p}}(x)$. Notons $A$ l'ensemble des idéaux premiers $\mathfrak{p}$ tels que $\alpha(\mathfrak{p})>0$ et $B$ l'ensemble des idéaux premiers $\mathfrak{p}$ tels que $\alpha(\mathfrak{p})<0$. Les décompositions des idéaux fractionnaires $x O_{K},\left(x^{2}+b\right) O_{K}$ et $\left(x^{2}-b\right) O_{K}$ en produit d'idéaux premiers sont de la forme:

$$
\begin{aligned}
x O_{K} & =\prod_{p \in A} p^{\alpha(\mathfrak{p})} \prod_{\mathfrak{p} \in B} \mathfrak{p}^{\alpha(\mathfrak{p})}, \\
\left(x^{2}+b\right) O_{K} & =\prod_{\mathfrak{p} \in B} \mathfrak{p}^{2 \alpha(\mathfrak{p})} \prod_{\{\mathfrak{q} ; \beta(\mathfrak{q})>0\}} \mathfrak{q}^{\beta(\mathfrak{q})}, \\
\left(x^{2}-b\right) O_{K} & =\prod_{\mathfrak{p} \in B} \mathfrak{p}^{2 \alpha(\mathfrak{p})} \prod_{\{\mathfrak{r} ; \gamma(\mathfrak{r})>0\}} \mathfrak{r}^{\gamma(\mathfrak{r})},
\end{aligned}
$$

où pour tous idéaux premiers q et $\mathfrak{r}$, on pose $\beta(\mathfrak{q})=v_{q}\left(x^{2}+b\right)$ et $\gamma(\mathfrak{r})=v_{\mathfrak{r}}\left(x^{2}-b\right)$. Démontrons le lemme suivant:

Lemme 4. Supposons que $P$ appartienne à $G$. Il existe un idéal $I$ de $O_{K}$ qui divise $8 O_{K}$ tel que l'on ait

$$
H_{x}(2 P)^{n}=\prod_{\{\mathfrak{q} ; \beta(\mathfrak{q})>0\} e} \mathrm{~N}(\mathfrak{q})^{2 \beta(\mathfrak{q})} \times \frac{1}{\mathrm{~N}(I)} .
$$

DÉMONSTRATION: D'après l'égalité (24), on a

$$
x(2 P) O_{K}=\prod_{\mathfrak{p} \in B} \mathfrak{p}^{\alpha(\mathfrak{p})} \prod_{\mathfrak{p} \in A} \mathfrak{p}^{-\alpha(\mathfrak{p})} \prod_{\{\mathfrak{q} ; \beta(\mathfrak{q})>0\}} \mathfrak{q}^{2 \beta(\mathfrak{q})} \prod_{\{\mathfrak{r} ; \gamma(\mathfrak{c})>0\}} \mathfrak{r}^{-\gamma(\mathfrak{r})}\left(4 O_{K}\right)^{-1} .
$$

Soit $I$ le plus grand commun diviseur des deux idéaux entiers

$$
\prod_{\{q ; \beta(q)>0\}} q^{2 \beta(q)} \text { et } \prod_{p \in A} p^{\alpha(p)} \prod_{p \in B} p^{-\alpha(p)} \prod_{\{\mathfrak{z}: \gamma(\mathfrak{p})>0\}} \mathfrak{r}^{\gamma(\mathfrak{r})}\left(4 O_{K}\right) \text {. }
$$

Le point $2 P$ vérifie l'hypothèse faite dans l'énoncé du Lemme 3 . L'égalité (28) et le Lemme 3 entraînent alors l'égalité

$$
H_{x}(2 P)^{n}=\prod_{\{q ; \beta(\mathfrak{q})>0\}} \mathrm{N}(\mathfrak{q})^{2 \beta(\mathfrak{q})} \times \frac{1}{\mathrm{~N}(I)} .
$$

Tout revient donc à prouver que

$$
I \text { divise } 8 O_{K} \text {. }
$$


Considérons pour cela un idéal premier $\mathfrak{P}$ de $O_{K}$ qui divise $I$. Puisque $\mathfrak{P}$ divise le produit des idéaux $q^{2 \beta(q)}$ où $\beta(q)>0$, on déduit de $(26)$ que

$$
\mathfrak{P} \text { ne divise pas } \prod_{\mathfrak{p} \in B} \mathfrak{p}^{-\alpha(\mathfrak{p})}
$$

Par suite, on a $v_{\mathfrak{p}}(x) \geqslant 0$. Il en résulte que

$$
v_{\mathfrak{P}}(x)=0
$$

En effet, dans le cas contraire, on aurait $v_{\mathfrak{P}}(x)>0$ et $v_{\mathfrak{P}}\left(x^{2}+b\right)>0$, d'où $v_{\mathfrak{p}}(b)>0$, ce qui, d'après le Lemme 2 , contredit le fait que $P$ soit dans $G$. On en déduit que

$$
I \text { divise } \prod_{\{r ; \gamma(r)>0\}} r^{\gamma(r)}\left(4 O_{K}\right) .
$$

En particulier, on a l'inégalité

$$
v_{\mathfrak{P}}\left(4\left(x^{2}-b\right)\right)>0
$$

Par ailleurs, on a

$$
v_{\mathfrak{P}}\left(x^{2}+b\right)>0
$$

Les conditions (30), (32) et (33) entraînent alors que $\mathfrak{P}$ est l'un des idéaux premiers de $O_{K}$ au-dessus de 2 . Il reste à démontrer que l'on a, $v_{\mathfrak{P}}(I)$ étant l'exposant de $\mathfrak{P}$ dans $I$,

$$
v_{\mathfrak{p}}(I) \leqslant 3 v_{\mathfrak{p}}(2)
$$

Supposons pour cela que l'on ait $v_{\mathfrak{p}}(I) \geqslant 3 v_{\mathfrak{p}}(2)+1$. D'après la condition (31) et le fait que $I$ divise le produit des idéaux $q^{2 \beta(q)}$ où $\beta(q)>0$, on a

$$
2 v_{\mathfrak{P}}\left(x^{2}+b\right) \geqslant v_{\mathfrak{P}}(I) \text { et } v_{\mathfrak{P}}\left(4\left(x^{2}-b\right)\right) \geqslant v_{\mathfrak{P}}(I) .
$$

On obtient alors

$$
v_{\mathfrak{P}}\left(x^{2}+b\right) \geqslant \frac{3 v_{\mathfrak{P}}(2)+1}{2} \text { et } \quad v_{\mathfrak{P}}\left(4\left(x^{2}-b\right)\right) \geqslant 3 v_{\mathfrak{P}}(2)+1,
$$

ce qui conduit aux inégalités

$$
v_{\mathfrak{P}}\left(x^{2}+b\right) \geqslant v_{\mathfrak{p}}(2)+1 \text { et } v_{\mathfrak{p}}\left(x^{2}-b\right) \geqslant v_{\mathfrak{p}}(2)+1
$$

On en déduit que $2 v_{\mathfrak{p}}(x) \geqslant 1$ et la condition (30) implique alors une contradiction. Cela prouve l'inégalité (34), puis la condition (29). D'où le résultat. 
2.5. Comparaison entre les haUteurs $\widehat{h}$ et $h_{x}$ SUR $\Gamma$. Notons $\widehat{h}$ la hauteur de . Véron-Tate sur $E_{b}$. On a une égalité de la forme $2 \widehat{h}=h_{x}+O(1)$ où $h_{x}=\log H_{x}$ (formules (19)). On va maintenant effectiviser cette égalité uniformément, c'est a dire indépendamment de $b$, sur le groupe $\Gamma$. Démontrons le résultat suivant:

Proposition 3. Soit $P$ un point de $\Gamma$. On a

$$
\left|2 \widehat{h}(P)-h_{x}(P)\right| \leqslant \log 2 .
$$

Prouvons pour cela l'énoncé ci-dessous:

Lemme 5. On a $\left|h_{x}(2 P)-4 h_{x}(P)\right| \leqslant 3 \log 2$.

DÉmonstration: Vérifions d'abord que cette inégalité est vraie si $2 P=O$. Tel est le cas si $P=O$, car alors $h_{x}(P)=h_{x}(2 P)=0$. Supposons $P \neq O$. Soit $a \in \overline{\mathbb{Q}}$ tel que $b=a^{2}$. On a alors $P=(0,0)$ ou bien $P=( \pm a, 0)$, cette dernière éventualité ne pouvant se produire que si $a$ est dans $K$. Pour tout plongement $\sigma: K \rightarrow \mathbb{R}$, le point $\sigma(P)$ étant dans $I_{\sigma}$, on a $P \neq(0,0)$, d'où $P=( \pm a, 0)$. Par ailleurs, $P$ appartient à $G$. D'après l'équivalence (20), l'élément $a$ est donc une unité de $O_{K}$. On obtient $H_{x}(P)=\left|N_{K / \mathrm{Q}}(a)\right|=1$, d'où $h_{x}(P)=0$ et notre assertion (car $h_{x}(2 P)=0$ ).

Supposons désormais $2 P \neq O$ et que les décompositions des idéaux fractionnaires $x O_{K}$ et $\left(x^{2}+b\right) O_{K}$ soient données par les formules (25) et (26).

Pour tout plongement $\sigma: K \rightarrow \mathbb{R}$, le point $\sigma(P)$ étant dans $I_{\sigma}$, on déduit de l'équivalence (21) que l'on a

$$
\mathrm{N}_{K / \mathbf{Q}}\left(x^{2}+b\right)=\prod_{\sigma}\left(\sigma\left(x^{2}\right)+\sigma(b)\right) \leqslant \mathrm{N}_{K / \mathbf{Q}}\left(2 x^{2}\right) .
$$

Il en résulte que

$$
\prod_{\mathfrak{p} \in B} \mathrm{~N}(\mathfrak{p})^{2 \alpha(\mathfrak{p})} \prod_{\{\mathfrak{q} ; \beta(\mathfrak{q})>0\}} \mathrm{N}(\mathfrak{q})^{\beta(\mathfrak{q})} \leqslant 2^{n} \prod_{\mathfrak{p} \in A} \mathrm{~N}(\mathfrak{p})^{2 \alpha(\mathfrak{p})} \prod_{\mathfrak{p} \in B} \mathrm{~N}(\mathfrak{p})^{2 \alpha(\mathfrak{p})},
$$

d'où l'inégalité

$$
\prod_{\{\mathfrak{q} ; \beta(\mathfrak{q})>0\}} \mathrm{N}(\mathfrak{q})^{\beta(\mathfrak{q})} \leqslant 2^{n} \prod_{\mathfrak{p} \in A} \mathrm{~N}(\mathfrak{p})^{2 \alpha(\mathfrak{p})} .
$$

On déduit alors des Lemmes 3 et 4 que l'on a

$$
H_{x}(2 P)^{n} \leqslant 2^{2 n} \prod_{p \in A} N(\mathfrak{p})^{4 \alpha(\mathfrak{p})}=2^{2 n} H_{x}(P)^{4 n} .
$$

Par ailleurs, $b$ étant totalement positif, on a

$$
\mathrm{N}_{K / \mathrm{Q}}\left(x^{2}+b\right) \geqslant \mathrm{N}_{K / \mathbf{Q}}\left(x^{2}\right) \text {. }
$$

D'après (26), on a donc

$$
\prod_{\mathfrak{p} \in B} \mathrm{~N}(\mathrm{p})^{2 \alpha(p)} \prod_{\{q ; \beta(q)>0\}} \mathrm{N}(q)^{\beta(q)} \geqslant \prod_{p \in A} N(p)^{2 \alpha(p)} \prod_{p \in B} N(p)^{2 \alpha(p)}
$$


autrement dit,

$$
\prod_{\{q ; \beta(q)>0\}} \mathrm{N}(\mathfrak{q})^{\beta(q)} \geqslant \prod_{\mathfrak{p} \in A} \mathrm{~N}(\mathfrak{p})^{2 \alpha(p)} .
$$

Puisque l'on a $\mathrm{N}\left(8 O_{K}\right)=8^{n}$, il résulte alors des Lemmes 3 et 4 l'inégalité

$$
H_{x}(2 P)^{n} \geqslant \frac{1}{8^{n}} H_{x}(P)^{4 n} .
$$

D'où le lemme en prenant les logarithmes des inégalités (35) et (36).

La Proposition 3 est une conséquence directe du Lemme 5 et du résultat qui suit (voir [7, p. 228-229]):

Lemme 6. Soit $S$ un sous-ensemble de $E_{b}(K)$ stable par multiplication par 2. Supposons qu'il existe une constante $c>0$ telle que pour tout $Q \in S$ on ait l'inégalité $\left|h_{x}(2 Q)-4 h_{x}(Q)\right| \leqslant c$. Alors, pour tout $Q \in S$, on a $\left|2 \widehat{h}(Q)-h_{x}(Q)\right| \leqslant c / 3$.

DÉmonstration: Rappelons que pour tout point $M \in E_{b}(K)$, on a (loco citato):

$$
\widehat{h}(M)=\frac{1}{2} \lim _{n \rightarrow+\infty} \frac{h_{x}\left(2^{n} M\right)}{4^{n}} .
$$

Considérons un point $Q \in S$. Pour tout entier $n \geqslant 1$ on a

$$
\frac{1}{4^{n}} h_{x}\left(2^{n} Q\right)-h_{x}(Q)=\sum_{j=0}^{n-1}\left(\frac{1}{4^{j+1}} h_{x}\left(2^{j+1} Q\right)-\frac{1}{4^{j}} h_{x}\left(2^{j} Q\right)\right) .
$$

On en déduit l'inégalité

$$
\left|\frac{1}{4^{n}} h_{x}\left(2^{n} Q\right)-h_{x}(Q)\right| \leqslant \sum_{j=0}^{n-1} \frac{1}{4^{j+1}}\left|h_{x}\left(2^{j+1} Q\right)-4 h_{x}\left(2^{j} Q\right)\right| .
$$

Par hypothèse, $2^{j} Q$ appartient à $S$. Il en résulte que l'on a

$$
\left|\frac{1}{4^{n}} h_{x}\left(2^{n} Q\right)-h_{x}(Q)\right| \leqslant c \sum_{j=0}^{n-1} \frac{1}{4^{j+1}} .
$$

On obtient ainsi

$$
\lim _{n \rightarrow+\infty}\left|\frac{1}{4^{n}} h_{x}\left(2^{n} Q\right)-h_{x}(Q)\right| \leqslant \frac{c}{3},
$$

ce qui entraîne le lemme.

Cela termine la démonstration de la Proposition 3. 
2.6. Hauteurs SuR LeS IMAgeS DE $C_{b}(K)$ DANS $E_{b}(K)$. Rappelons que l'on dispose de deux morphismes $\phi_{b}: C_{b} \rightarrow E_{b}$ et $\psi_{b}: C_{b} \rightarrow E_{b}$ définis sur $K$ par les égalités

et

$$
\begin{aligned}
& \phi_{b}([x, y, z])=\left[-x^{2} z, x y^{2}, z^{3}\right] \\
& \psi_{b}([x, y, z])=\left[-y^{2} z, y x^{2}, z^{3}\right] .
\end{aligned}
$$

Considérons dans tout ce paragraphe un point $Q=[x, y, z] \in C_{b}(K)$. On a $z \neq 0$, car sinon -1 est une puissance quatrième dans $K$, ce qui contredit le fait que $K$ soit totalement réel. On peut donc supposer que l'on a $z=1$. On a ainsi l'égalité

$$
x^{4}+y^{4}=b .
$$

Posons $P_{1}=\phi_{b}(Q)$ et $P_{2}=\psi_{b}(Q)$ dans $E_{b}(K)$. On a dans le modèle affine (16)

$$
P_{1}=\left(-x^{2}, x y^{2}\right) \quad \text { et } \quad P_{2}=\left(-y^{2}, y x^{2}\right) .
$$

Lemme 7. Les points $P_{1}$ et $P_{2}$ appartiennent à $G$.

DÉmonstration: Soit $v$ une place finie de $K$ telle que $v(b)>0$. D'après l'équivalence (20) et les égalités (38), il s'agit de démontrer que l'on a $v(x) \leqslant 0$. Supposons $v(x)>0$. L'égalité (37) entraîne alors $v(y)>0$ puis $v(b) \geqslant 4$, ce qui contredit la condition 1 de l'énoncé du Théorème 3 . D'où notre assertion et le résultat.

Proposition 4. On a les inégalités

$$
\left|h_{x}\left(2 P_{1}\right)-h_{x}\left(2 P_{2}\right)\right| \leqslant 5 \log 2 \quad \text { et } \quad\left|\widehat{h}\left(P_{1}\right)-\widehat{h}\left(P_{2}\right)\right| \leqslant \frac{7}{8} \log 2 .
$$

DÉmonstration: (1) Prouvons la première inégalité. Elle est vraie si $x y=0$, car dans ce cas, compte tenu de l'égalité (37), les points $P_{1}$ et $P_{2}$ sont d'ordre 2 dans $E_{b}(K)$. Supposons désormais $x y$ non nul. Dans ce cas, $P_{1}$ et $P_{2}$ ne sont pas d'ordre 2 et les abscisses de $2 P_{1}$ et $2 P_{2}$ sont données par les égalités

$$
x\left(2 P_{1}\right)=\frac{\left(x^{4}+b\right)^{2}}{4 x^{2}\left(b-x^{4}\right)} \quad \text { et } \quad x\left(2 P_{2}\right)=\frac{\left(y^{4}+b\right)^{2}}{4 y^{2}\left(b-y^{4}\right)} .
$$

Pour tout idéal premier $\mathfrak{p}$ de $O_{K}$, posons, comme précédemment, $\alpha_{\mathfrak{p}}=v_{\mathfrak{p}}(x)$ et notons $A$ l'ensemble des idéaux premiers $\mathfrak{p}$ tels que $v_{\mathrm{p}}(x)>0$ et $B$ l'ensemble des idéaux premiers tels que $v_{\mathrm{p}}(x)<0$. Les décompositions des idéaux fractionnaires $x O_{K}$ et $\left(x^{4}+b\right) O_{K}$ en produit d'idéaux premiers sont de la forme:

$$
\begin{aligned}
x O_{K} & =\prod_{p \in A} p^{\alpha(p)} \prod_{p \in B} p^{\alpha(p)}, \\
\left(x^{4}+b\right) O_{K} & =\prod_{p \in B} p^{4 \alpha(p)} \prod_{\{q ; \beta(q)>0\}} q^{\beta(q)} .
\end{aligned}
$$


Par ailleurs, pour tout idéal premier $\mathfrak{p}$ de $O_{K}$, compte tenu de $(37)$, on a $v_{\mathfrak{p}}(x)<0$ si et seulement si $v_{p}(y)<0$ et dans ce cas $v_{p}(x)=v_{p}(y)$. Il en résulte que la décomposition de $\left(y^{4}+b\right) O_{K}$ en produit d'idéaux premiers est de la forme suivante:

$$
\left(y^{4}+b\right) O_{K}=\prod_{\mathfrak{p} \in B} \mathfrak{p}^{4 \alpha(\mathfrak{p})} \prod_{\{\mathfrak{r} ; \gamma(\mathfrak{r})>0\}} \mathfrak{r}^{\gamma(\mathfrak{r})}
$$

D'après les Lemmes 4 et 7 et les égalités (38), il existe ainsi deux idéaux $J_{1}$ et $J_{2}$ de $O_{K}$ qui divisent $8 O_{K}$ tels que l'on ait

$$
\begin{aligned}
& H_{x}\left(2 P_{1}\right)^{n}=\prod_{\{\mathfrak{q} ; \beta(\mathfrak{q})>0\}} \mathrm{N}(\mathrm{q})^{2 \beta(\mathrm{q})} \times \frac{1}{\mathrm{~N}\left(J_{1}\right)}, \\
& H_{x}\left(2 P_{2}\right)^{n}=\prod_{\{\mathrm{r} ; \gamma(\mathrm{r})>0\}} \mathrm{N}(\mathrm{r})^{2 \gamma(\mathrm{r})} \times \frac{1}{\mathrm{~N}\left(J_{2}\right)} .
\end{aligned}
$$

D'après (40) et (41) on obtient donc l'égalité

$$
\frac{H_{x}\left(2 P_{1}\right)^{n}}{H_{x}\left(2 P_{2}\right)^{n}}=\frac{\mathrm{N}_{K / \mathrm{Q}}\left(x^{4}+b\right)^{2}}{\mathrm{~N}_{K / \mathrm{Q}}\left(y^{4}+b\right)^{2}} \times \frac{N\left(J_{2}\right)}{N\left(J_{1}\right)} .
$$

L'égalité (37) entraîne alors

$$
\frac{H_{x}\left(2 P_{1}\right)^{n}}{H_{x}\left(2 P_{2}\right)^{n}}=\frac{\mathrm{N}_{K / \mathrm{Q}}\left(2 x^{4}+y^{4}\right)^{2}}{\mathrm{~N}_{K / \mathrm{Q}}\left(2 y^{4}+x^{4}\right)^{2}} \times \frac{N\left(J_{2}\right)}{N\left(J_{1}\right)}
$$

Posons

$$
t=\frac{x^{4}}{y^{4}}
$$

On obtient

$$
\frac{H_{x}\left(2 P_{1}\right)^{n}}{H_{x}\left(2 P_{2}\right)^{n}}=\frac{\mathrm{N}_{K / \mathrm{Q}}(2 t+1)^{2}}{\mathrm{~N}_{K / \mathrm{Q}}(t+2)^{2}} \times \frac{N\left(J_{2}\right)}{N\left(J_{1}\right)}
$$

ce qui conduit à

$$
\frac{H_{x}\left(2 P_{1}\right)^{n}}{H_{x}\left(2 P_{2}\right)^{n}}=\mathrm{N}_{K / \mathrm{Q}}\left(2-\frac{3}{t+2}\right)^{2} \times \frac{N\left(J_{2}\right)}{N\left(J_{1}\right)} .
$$

Pour tout plongement $\sigma: K \rightarrow \mathbb{R}$, on a $\sigma(t) \geqslant 0$, d'où il résulte que l'on a

$$
\frac{1}{2} \leqslant 2-\frac{3}{\sigma(t)+2} \leqslant 2 \text {. }
$$

On en déduit les inégalités

$$
\frac{1}{2^{n}} \leqslant N_{K / Q}\left(2-\frac{3}{t+2}\right) \leqslant 2^{n}
$$

On déduit alors de (42) et (43) que l'on a

$$
\frac{1}{2^{2 n}} \times \frac{1}{8^{n}}=\frac{1}{2^{5 n}} \leqslant \frac{H_{x}\left(2 P_{1}\right)^{n}}{H_{x}\left(2 P_{2}\right)^{n}} \leqslant 2^{2 n} \times 8^{n}=2^{5 n}
$$


D'où la première inégalité de la proposition.

(2) En ce qui concerne la deuxième inégalité, on a

$$
\left|\widehat{h}\left(P_{1}\right)-\widehat{h}\left(P_{2}\right)\right|=\frac{1}{4}\left|\widehat{h}\left(2 P_{1}\right)-\widehat{h}\left(2 P_{2}\right)\right| .
$$

Il en résulte que

$$
\begin{aligned}
& \left|\widehat{h}\left(P_{1}\right)-\widehat{h}\left(P_{2}\right)\right| \\
& \quad \leqslant \frac{1}{4}\left(\left|\widehat{h}\left(2 P_{1}\right)-\frac{1}{2} h_{x}\left(2 P_{1}\right)\right|+\frac{1}{2}\left|h_{x}\left(2 P_{1}\right)-h_{x}\left(2 P_{2}\right)\right|+\left|\frac{1}{2} h_{x}\left(2 P_{2}\right)-\widehat{h}\left(2 P_{2}\right)\right|\right) .
\end{aligned}
$$

Par ailleurs, on déduit du Lemme 7 que $2 P_{1}$ et $2 P_{2}$ appartiennent au sous-groupe $\Gamma$ de $E_{b}(K)$. L'inégalité déjà prouvée et la Proposition 3 entraînent alors le résultat. D'où la Proposition.

2.7. Fin de la démonstration du ThÉorème 3. On suppose que l'ensemble $C_{b}(K)$ est non vide. Il s'agit d'obtenir une contradiction. Comme on l'a constaté au début du paragraphe 2.6, il existe alors $Q=(x, y) \in C_{b}(K)$ dans l'ouvert affine $z=1$, l'égalité (37) étant ainsi satisfaite. On pose comme précédemment

$$
P_{1}=\phi_{b}(Q) \quad \text { et } \quad P_{2}=\psi_{b}(Q)
$$

Vérifions que l'on a

$$
P_{1}+P_{2} \neq O \quad \text { et } \quad P_{1}-P_{2} \neq O \text {. }
$$

Supposons $P_{1}= \pm P_{2}$. D'après (38), on a $P_{1}=\left(-x^{2}, x y^{2}\right)$ et $P_{2}=\left(-y^{2}, y x^{2}\right)$, d'où $x^{2}=y^{2}$, puis $b=2 x^{4}$. Soit $v$ une place finie de $K$ telle que $v(x) \neq 0$. On a $v(2)>0$ : en effet, dans le cas contraire, on aurait $v(b)=4 v(x)>0$ (car $b \in O_{K}$ ) ce qui contredit la condition 1 de l'énoncé du Théorème 3. Par suite, on a

$$
x O_{K}=\prod_{\mathfrak{p} \mid 2} \mathfrak{p}^{\alpha(\mathfrak{p})},
$$

où p parcourt l'ensemble des idéaux premiers de $O_{K}$ au-dessus de 2 et où $\alpha(\mathfrak{p}) \in \mathbb{Z}$. Pour la même raison, l'égalité $b=2 x^{4}$ entraîne $\alpha(\mathfrak{p}) \leqslant 0$ pour tout $\mathfrak{p}$. On en déduit que

$$
\mathrm{N}_{K / \mathrm{Q}}(b) \leqslant 2^{n}
$$

ce qui contredit l'inégalité $\mathrm{N}_{K / \mathbf{Q}}(b) \geqslant 2^{(11 n) / 2}$ intervenant dans la condition 3 de l'énoncé du théorème. D'où l'assertion (44).

On désigne par $R$ un point de $E_{b}(K)$ vérifiant la condition suivante: si $E_{b}(K)$ est de rang 0 on a $R=O$, et si $E_{b}(K)$ est de rang 1 alors $R$ est un générateur de $E_{b}(K)$ modulo 
son sous-groupe de torsion. Il existe ainsi des entiers $m, n$ et des points de torsion $T_{1}, T_{2}$ de $E_{b}(K)$ tels que l'on ait

$$
P_{1}=m R+T_{1} \quad \text { et } \quad P_{2}=n R+T_{2}
$$

Soit $N$ un multiple des ordres de $T_{1}$ et $T_{2}$. On a $N P_{1}=N m R$ et $N P_{2}=N n R$. On en déduit les égalités

$$
\widehat{h}\left(P_{1}\right)=m^{2} \widehat{h}(R) \quad \text { et } \quad \widehat{h}\left(P_{2}\right)=n^{2} \widehat{h}(R)
$$

De même, on a

$$
\widehat{h}\left(P_{1}+P_{2}\right)=(m+n)^{2} \widehat{h}(R) \quad \text { et } \quad \widehat{h}\left(P_{1}-P_{2}\right)=(m-n)^{2} \widehat{h}(R) .
$$

On en déduit que

$$
\begin{array}{lll}
\widehat{h}\left(P_{1}+P_{2}\right) \leqslant\left|m^{2}-n^{2}\right| \widehat{h}(R) & \text { si } & m n \leqslant 0, \\
\widehat{h}\left(P_{1}-P_{2}\right) \leqslant\left|m^{2}-n^{2}\right| \widehat{h}(R) & \text { si } & m n \geqslant 0 .
\end{array}
$$

Considérons alors un plongement $\sigma: K \rightarrow \mathbb{R}$. D'après (38), on a

$$
\sigma\left(x\left(P_{1}\right)\right) \leqslant 0 \quad \text { et } \quad \sigma\left(x\left(P_{2}\right)\right) \leqslant 0
$$

par suite $\sigma\left(P_{1}\right)$ et $\sigma\left(P_{2}\right)$ n'appartiennent pas à la composante neutre $I_{\sigma}$. Par définition de la loi de groupe sur $E_{\sigma(b)}$, les points $\sigma\left(P_{1}+P_{2}\right)$ et $\sigma\left(P_{1}-P_{2}\right)$ sont donc dans $I_{\sigma}$. D'après le Lemme 7 , il en résulte que

$$
P_{1}+P_{2} \in \Gamma \quad \text { et } \quad P_{1}-P_{2} \in \Gamma .
$$

D'après la Proposition 3, on obtient alors

$$
\begin{aligned}
& h_{x}\left(P_{1}+P_{2}\right) \leqslant 2 \hat{h}\left(P_{1}+P_{2}\right)+\log 2 \\
& h_{x}\left(P_{1}-P_{2}\right) \leqslant 2 \hat{h}\left(P_{1}-P_{2}\right)+\log 2 .
\end{aligned}
$$

Compte tenu des conditions (46), (47) et (48), on a donc les inégalités

$$
\begin{array}{lll}
h_{x}\left(P_{1}+P_{2}\right) \leqslant 2\left|m^{2}-n^{2}\right| \widehat{h}(R)+\log 2 & \text { si } & m n \leqslant 0, \\
h_{x}\left(P_{1}-P_{2}\right) \leqslant 2\left|m^{2}-n^{2}\right| \widehat{h}(R)+\log 2 & \text { si } & m n \geqslant 0 .
\end{array}
$$

Supposons $m n \leqslant 0$. Dans ce cas, les égalités (45) entraînent

$$
h_{x}\left(P_{1}+P_{2}\right) \leqslant 2\left|\widehat{h}\left(P_{1}\right)-\widehat{h}\left(P_{2}\right)\right|+\log 2
$$

D'après la Proposition 4, on en déduit que

$$
h_{x}\left(P_{1}+P_{2}\right) \leqslant \frac{11}{4} \log 2 .
$$


De même, si $m n \geqslant 0$, on obtient

$$
h_{x}\left(P_{1}-P_{2}\right) \leqslant \frac{11}{4} \log 2 .
$$

Démontrons maintenant que l'on a

$$
n h_{x}\left(P_{1}+P_{2}\right) \geqslant \frac{\log \mathrm{N}_{K / \mathrm{Q}}(b)}{2} \text { et } n h_{x}\left(P_{1}-P_{2}\right) \geqslant \frac{\log \mathrm{N}_{K / \mathrm{Q}}(b)}{2} .
$$

D'après (44), le point $P_{1}+P_{2}$ est non nul. Posons $P_{1}+P_{2}=(u, w) \in E_{b}(K)$. On a

$$
H_{x}\left(P_{1}+P_{2}\right)^{n}=\prod_{v \in M_{K}} \operatorname{Max}\left(1,|u|_{v}\right)^{n_{v}} \geqslant \prod_{v \in M_{K}^{\infty}} \operatorname{Max}\left(1,|u|_{v}\right) .
$$

Par ailleurs, puisque $P_{1}+P_{2}$ appartient à $\Gamma$, on a $|\sigma(u)|=\sigma(u) \geqslant \sqrt{\sigma(b)}$ pour tout plongement $\sigma: K \rightarrow \mathbb{R}$. On a ainsi

$$
H_{x}\left(P_{1}+P_{2}\right)^{n} \geqslant \prod_{\sigma} \operatorname{Max}(1, \sqrt{\sigma(b)})
$$

D'après la condition (15), pour tout $\sigma$ on a $\sigma(b) \geqslant 1$. On en déduit que

$$
H_{x}\left(P_{1}+P_{2}\right)^{n} \geqslant \sqrt{\mathrm{N}_{K / \mathbf{Q}}(b)}
$$

d'où la première inégalité de (51). La démonstration de la deuxième inégalité de (51) est la même. Les conditions (49) et (50) impliquent alors

$$
\log N_{K / \mathbf{Q}}(b) \leqslant \frac{11 n}{2} \log 2
$$

d'où

$$
N K / Q(b) \leqslant 2^{(11 n) / 2} .
$$

La première inégalité de (13) conduit alors à une contradiction.

Cela termine la démonstration du Théorème 3 .

APPENDICE 1 - EXEMPLE D'EFFECTIVITÉ DU THÉORÈME DES ZÉROS DE HILBERT

On est confronté dans la démonstration de la Proposition 1 au problème de l'effectivité du théorème des zéros de Hilbert dans un cas particulier. Considérons trois indéterminées $X, Y$ et $Z$. Soit $I$ l'idéal homogène de l'anneau $\mathbb{C}[X, Y, Z]$ engendré par les polynômes $F, G$ et $H$ suivants:

$$
F=X^{4}+Y^{4}-Z^{4}, \quad G=\left(X^{2}+X Y+Y^{2}\right)^{2} \quad \text { et } \quad H=(X+Y)^{2} Z^{2} .
$$

Soit $V_{p}(I)$ l'ensemble algébrique projectif de $\mathbb{P}^{2}$ associé à $I$. Vérifions que $V_{p}(I)$ est vide. Supposons qu'il existe un point $[x, y, z] \in V_{p}(I)$. On a $(x+y) z=0$. Si $z=0$, alors $x y \neq 0$ 
et il existe $\alpha \in \mathbb{C}$ tel que $x=\alpha y$ avec $\alpha^{4}=-1$. L'égalité $x^{2}+x y+y^{2}=0$ conduit alors à $1+\alpha+\alpha^{2}=0$, d'où $\alpha^{3}=1$ et une contradiction. Si $x+y=0$, l'égalité $x^{2}+x y+y^{2}=0$ entraîne $x=y=0$, d'où de nouveau une contradiction et notre assertion. D'après le théorème des zéros, il existe donc un entier $n \geqslant 1$ tel que les monômes $X^{n}, Y^{n}$ et $Z^{n}$ appartiennent à $I$. Il s'agit ici d'effectiviser cette condition. On a l'énoncé suivant:

Proposition. L'entier $n=12$ convient. Posons

$$
\begin{gathered}
P=3 X^{8}+4 Y^{2} X^{6}+4 Y^{4} X^{4}+2 Y^{6} X^{2}+Y^{8} \\
Q=-2 X^{8}+4 Y X^{7}-6 Y^{2} X^{6}+4 Y^{3} X^{5}+\left(10 Z^{4}-3 Y^{4}\right) X^{4}+2 Y^{5} X^{3} \\
-3 Y^{6} X^{2}+2 Y^{7} X-Y^{8}+4 Z^{4} Y^{4} \\
R=-7 Z^{2} X^{6}-6 Z^{2} Y X^{5}-7 Z^{2} Y^{2} X^{4}-3 Z^{2} Y^{4} X^{2}-2 Z^{2} Y^{5} X-3 Z^{2} Y^{6} .
\end{gathered}
$$

On a les égalités

$$
\begin{aligned}
Z^{6} & =-Z^{2} F+2 Z^{2} G-(X+Y)^{2} H, \\
X^{12} & =P F+Q G+R H \quad \text { et } \quad Y^{12}=\widetilde{P} F+\widetilde{Q} G+\widetilde{R} H,
\end{aligned}
$$

où $\widetilde{P}(X, Y, Z)=P(Y, X, Z), \widetilde{Q}(X, Y, Z)=Q(Y, X, Z)$ et $\widetilde{R}(X, Y, Z)=R(Y, X, Z)$.

DÉMONSTRATion: Il est immédiat de vérifier les égalités annoncées à l'aide d'un. ordinateur. On est en fait parvenu à ce résultat en partant de l'égalité

$$
Z^{4}+(X+Y)^{4}=2 G-F
$$

L'égalité (1) en résulte. Par ailleurs, on déduit de (3) les deux égalités

$$
\begin{aligned}
G & =Z^{4}+F+2 X Y(X+Y)^{2}-(X Y)^{2} \\
(X Y)^{2}(X+Y)^{4} & =-(X Y)^{2} F+\left(2 X^{2} Y^{2}-Z^{4}\right) G+Z^{2}\left(X^{2}+Y^{2}\right) H .
\end{aligned}
$$

Elles entraînent que $(X Y)^{4}$ appartient à $I$, puis que

$$
\begin{aligned}
(X Y)^{4} & =S F+T G+U H, \\
\text { avec } \quad S & =-\left(X^{2}+Y^{2}\right)^{2}, \quad T=X^{4}-2 Y X^{3}+3 Y^{2} X^{2}-2 Y^{3} X+Y^{4}-4 Z^{4}, \\
U & =3(X Z)^{2}+2 X Y Z^{2}+3(Y Z)^{2} .
\end{aligned}
$$

En considérant alors l'égalité

$$
X^{8}+Y^{8}=Z^{8}+F^{2}+2 Z^{4} F-2(X Y)^{4},
$$

et en multipliant ses deux membres par $X^{4}$ (respectivement $Y^{4}$ ) on obtient la première (respectivement la deuxième) égalité de (2). 


\section{APPENDICE 2 - TORSION GALOISIENNE}

L'objectif de cet appendice est de rappeler un résultat de Silverman concernant les tordues galoisiennes de courbes que l'on utilise dans la démonstration du Théorème 1 (voir [6]). Les courbes intervenant ci-dessous sont implicitement supposées projectives et lisses et plongées dans un même espace projectif $\mathbb{P}^{n}$. Tous les corps considérés sont par ailleurs contenus dans $\overline{\mathbb{Q}}$.

Considérons un corps de nombres $K$ et une courbe $\mathscr{C}$ définie sur $K$. Soit $\mathscr{C}$ une courbe définie sur $K$ isomorphe à $\mathscr{C}$ sur une extension finie $L$ de $K$. On suppose que $L$ est minimale au sens où si $M$ est un sous-corps strict de $L$, les courbes $\mathscr{C}$ et $\mathscr{C}$ ne sont pas isomorphes sur $M$. Notons:

(a) $G_{K}$ le groupe de Galois de $\overline{\mathbb{Q}}$ sur $K$.

(b) $d$ le degré de $L$ sur $K$.

(c) $\delta_{K}$ le nombre de places archimédiennes de $K$.

(d) $D_{L / K}$ le discriminant relatif de l'extension $L / K$.

(e) $h: \mathbb{P}^{n}(\overline{\mathbb{Q}}) \rightarrow \mathbb{R}$ la hauteur absolue logarithmique ([7, p. 215]); elle est définie par une formule analogue à celle si $n=2$ (paragraphe 1.2).

Soit $f: \mathscr{C}^{\prime} \rightarrow \mathscr{C}$ un isomorphisme défini sur $L$ de $\mathscr{C}^{\prime}$ sur $\mathscr{C}$. Pour tout $\sigma \in G_{K}$, on pose

$$
\xi(\sigma)={ }^{\sigma} f \circ f^{-1} \in \operatorname{Aut}(\mathscr{C}) .
$$

THÉoRème. Soit $Q$ un point de $f\left(\mathscr{C}^{\prime}(K)\right)$. On est dans l'un des cas suivants:

(1) il existe $\sigma \in G_{K}$ tel que $\xi(\sigma)$ ne soit pas l'identité de $\mathscr{C}$ et que $Q=\xi(\sigma)(Q)$.

(2) On a l'inégalité

$$
\log N_{K / \mathbf{Q}} D_{L / K} \leqslant\left(2(d-1) h(Q)+\delta_{K} \log d\right) d
$$

DÉmonstration: On prouve d'abord le lemme suivant.

LEMME. L'ensemble $f\left(\mathscr{C}^{\prime}(K)\right)$ est formé des points $A \in \mathscr{C}(L)$ tels que, pour tout $\sigma$ dans $G_{K}$, on ait ${ }^{\sigma} A=\xi(\sigma)(A)$.

DÉmonstration: Pour tout point $P \in \mathscr{C}^{\prime}(\overline{\mathbb{Q}})$ et tout $\sigma \in G_{K}$, on a

$$
{ }^{\sigma}(f(P))=\xi(\sigma)\left(f\left({ }^{\sigma} P\right)\right) .
$$

Cela entraine que $f\left(\mathscr{C}^{\prime}(K)\right)$ satisfait la condition du lemme. Inversement, soit $A \in \mathscr{C}(L)$ tel que, pour tout $\sigma$ dans $G_{K}$, on ait ${ }^{\sigma} A=\xi(\sigma)(A)$. Posons $P=f^{-1}(A)$. D'après (2), on a les égalités

$$
\xi(\sigma)\left(f\left({ }^{\sigma} P\right)\right)={ }^{\sigma} A=\xi(\sigma)(A)=\xi(\sigma)(f(P)) .
$$


On en déduit que $f\left({ }^{\sigma} P\right)=f(P)$, puis que $P={ }^{\circ} P$. Il en résulte que $P$ appartient à $\mathscr{C}^{\prime}(K)$, autrement dit que $A$ est dans $f\left(\mathscr{C}^{\prime}(K)\right)$. D'où le lemme.

Le théorème se déduit comme suit. Soit $K(Q)$ le sous-corps de $\overline{\mathbb{Q}}$ engendré par $K$ et les coordonnées de $Q$ dans $\mathbb{P}^{n}(\overline{\mathbb{Q}})$. On a l'inclusion

$$
K(Q) \subseteq L
$$

Supposons que la condition 1 du théorème ne soit pas réalisée. Soit $\sigma$ un élément de $\operatorname{Gal}(\overline{\mathbb{Q}} / K(Q))$. On a ${ }^{\sigma} Q=Q$ et on déduit du lemme l'égalité

$$
Q=\xi(\sigma)(Q)
$$

D'après l'hypothèse faite, $\xi(\sigma)$ est donc l'automorphisme identité de $\mathscr{E}$, autrement dit $f$ est définie sur $K(Q)$. Les courbes $\mathscr{C}$ et $\mathscr{L}^{\prime}$ sont donc isomorphes sur $K(Q)$. D'après l'inclusion (3) et le caractère minimal de $L$, on a donc $L=K(Q)$. Le Théorème 2 de [6] entraîne alors directement l'inégalité (1). D'où le résultat.

APPENDICE 3 - SUR LES ENTIERS TOTALEMENT POSITIFS D'UN CORPS TOTALEMENT RÉEL

Soit $K$ un corps de nombres totalement réel de degré $n$ sur $\mathbb{Q}$, d'anneau d'entiers $O_{K}$. Soient $\sigma_{1}, \ldots, \sigma_{n}$ les $n$ plongements de $K$ dans $\mathbb{R}$. Pour tout $x \in O_{K}$ on note $\mathrm{N}_{K / \mathbf{Q}}(x)$ sa norme de $K$ sur $\mathbb{Q}$ et $H(x)$ la hauteur de $x$ relative à $K$. On a

$$
H(x)=\prod_{i=1}^{n} \operatorname{Max}\left(1,\left|\sigma_{i}(x)\right|\right)
$$

On utilise dans la démonstration du Théorème 3 le résultat ci-dessous concernant les entiers totalement positifs de $K$.

Proposition. Soit $\left(u_{1}, \ldots, u_{n-1}\right)$ un système d'unités fondamentales de $O_{K}$. Soit $b$ un élément de $O_{K}$ tel que l'on ait $\sigma_{j}(b)>0$ pour tout $j=1, \ldots, n$. Supposons que $b$ vérifie la condition suivante:

$$
\mathrm{N}_{K / \mathrm{Q}}(b) \geqslant\left(\prod_{k=1}^{n-1} H\left(u_{k}\right)\right)^{4}
$$

Alors, il existe une unité $u$ de $O_{K}$ telle que l'on ait

$$
\sigma_{j}\left(b u^{4}\right) \geqslant 1 \text { pour } j=1, \ldots, n .
$$

DÉmonstration: Soit $L: K^{*} \rightarrow \mathbb{R}^{n}$ le plongement logarithmique de $K^{*}$, c'est a dire l'homomorphisme de groupes défini pour tout $x \in K^{*}$ par

$$
L(x)=\left(\log \left(\left|\sigma_{1}(x)\right|\right), \ldots, \log \left(\left|\sigma_{n}(x)\right|\right)\right) .
$$


L'image par $L$ du groupe des unités de $O_{K}$ est un réseau $\Lambda$ de l'hyperplan $V$ de $\mathbb{R}^{n}$ d'équation $x_{1}+\cdots+x_{n}=0$. Posons

$$
e_{k}=L\left(u_{k}\right) \text { pour } \quad k=1, \ldots, n-1
$$

Le système $\left(e_{1}, \ldots, e_{n-1}\right)$ est une base de $\Lambda$ sur $\mathbb{Z}$ donc une base de $V$ sur $\mathbb{R}$. Posons par ailleurs

$$
\beta=L(b), \quad \beta=\left(\beta_{1}, \ldots, \beta_{n}\right) \quad \text { et } \quad S=\beta_{1}+\cdots+\beta_{n} .
$$

Puisque $b$ est totalement positif, on a donc

$$
\beta_{j}=\log \left(\sigma_{j}(b)\right) \text { pour } j=1, \ldots, n \text {. }
$$

Munissons $\mathbb{R}^{n}$ de la norme définie pour tout $\left(x_{1}, \ldots, x_{n}\right) \in \mathbb{R}^{n}$ par

$$
\left\|\left(x_{1}, \ldots, x_{n}\right)\right\|=\sum_{h=1}^{n}\left|x_{h}\right| .
$$

Vérifions que l'on a

$$
S \geqslant 2 \sum_{k=1}^{n-1}\left\|e_{k}\right\|
$$

Considérons pour cela une unité $a$ de $O_{K}$. Posons

$$
P=\prod_{i}\left|\sigma_{i}(a)\right| \quad \text { et } \quad P^{\prime}=\prod_{j}\left|\sigma_{j}(a)\right|,
$$

où $i$ parcourt l'ensemble des indices pour lesquels $\left|\sigma_{i}(a)\right| \geqslant 1$ et $j$ parcourt l'ensemble des indices pour lesquels $\left|\sigma_{j}(a)\right|<1$. On a $P P^{\prime}=1$. Il en résulte que

$$
\begin{aligned}
\sum_{h=1}^{n}\left|\log \left(\left|\sigma_{h}(a)\right|\right)\right|=\sum_{i} & \log \left(\left|\sigma_{h}(a)\right|\right) \\
& -\sum_{j} \log \left(\left|\sigma_{h}(a)\right|\right)=\log (P)-\log \left(P^{\prime}\right)=2 \log (P) .
\end{aligned}
$$

Par ailleurs, d'après la formule (1), on a $H(a)=P$. D'après le calcul précédent, on a donc

$$
\|L(a)\|=2 \log (H(a))
$$

L'inégalité (2) se traduit alors, en prenant les logarithmes, par la condition (4).

Soit $M$ la maille du réseau $\Lambda$ formée des combinaisons linéaires

$$
\sum_{k=1}^{n-1} x_{k} e_{k}
$$


où les coefficients $x_{k}$ décrivent l'intervalle $[-1 / 2,1 / 2]$. Pour tout $i=1, \ldots, n$, soit $\left(e_{k}\right)_{i}$ la $i$-ème coordonnée de $e_{k}$ dans la base canonique de $\mathbb{R}^{n}$. Pour tout $z=\left(z_{1}, \ldots, z_{n}\right) \in M$, on a les inégalités

$$
2\left|z_{i}\right| \leqslant \sum_{k=1}^{n-1}\left|\left(e_{k}\right)_{i}\right|:=\ell_{i} .
$$

Pour tout $i=1, \ldots, n$, le réseau $\Lambda$ n'étant pas contenu dans l'hyperplan de $\mathbb{R}^{n}$ d'équation $x_{i}=0$, on a $\ell_{i}>0$. Posons alors

$$
w_{i}=\frac{\ell_{i}}{\ell_{1}+\cdots+\ell_{n}} \quad \text { et } \quad w=\left(w_{1}, \ldots, w_{n}\right) \in \mathbb{R}^{n} .
$$

Considérons le vecteur

$$
y=\frac{1}{4}(S w-\beta) .
$$

Par définition, $y$ appartient à $V$. Il existe donc un élément $\lambda=\left(\lambda_{1}, \ldots, \lambda_{n}\right) \in \Lambda$ tel que $y-\lambda$ soit dans $M$. On déduit alors de (5) que l'on a

$$
\beta_{i}+4 \lambda_{i} \geqslant S w_{i}-2 \ell_{i} \text { pour } i=1, \ldots, n .
$$

Il existe des entiers $t_{k} \in \mathbb{Z}$ tels que l'on ait

$$
\lambda=\sum_{k=1}^{n-1} t_{k} e_{k}
$$

Posons

$$
u=\prod_{k=1}^{n-1} u_{k}^{t_{k}}
$$

Vérifions que l'unité $u$ de $O_{K}$ satisfait la conclusion de la proposition. On a $L(u)=\lambda$, d'où $\lambda_{i}=\log \left(\left|\sigma_{i}(u)\right|\right)$. Compte tenu de (3), les inégalités (6) s'écrivent donc

$$
\log \left(\sigma_{i}(b)\right)+4 \log \left(\left|\sigma_{i}(u)\right|\right) \geqslant S w_{i}-2 \ell_{i}
$$

autrement dit,

$$
\sigma_{i}\left(b u^{4}\right) \geqslant \exp \left(S w_{i}-2 \ell_{i}\right) \text { pour } i=1, \ldots, n .
$$

Par ailleurs, on a

$$
\ell_{1}+\cdots+\ell_{n}=\sum_{k=1}^{n-1} \sum_{i=1}^{n}\left|\left(e_{k}\right)_{i}\right|=\sum_{k=1}^{n-1}\left\|e_{k}\right\| .
$$

Il résulte alors de la condition (4) que l'on a

$$
S \geqslant 2 \operatorname{Max}\left(\frac{\ell_{1}}{w_{1}}, \ldots, \frac{\ell_{n}}{w_{n}}\right) .
$$

D'après $(7)$, on obtient ainsi que $\sigma_{i}\left(b u^{4}\right) \geqslant 1$ pour tout $i=1, \ldots, n$. D'où la proposition. 


\section{REFERENCES}

[1] J.E. Cremona, M. Prickett et S. Siksek, 'Height difference bounds for elliptic curves over number fields', J. Number Theory 116 (2006), 42-68.

[2] V.A. Dem'janenko, 'The Indeterminate Equations $x^{6}+y^{6}=a z^{2}, x^{6}+y^{6}=a z^{3}$, $x^{4}+y^{4}=a z^{4}$, Amer. Math. Soc. Transl. 119 (1983), 27-34.

[3] G. Grigorov et J. Rizov, 'Heights on elliptic curves and the diophantine equation $x^{4}+y^{4}=c z^{4}$, (preprint), Sophia University (1998).

[4] A. Kraus, 'Quelques remarques à propos des invariants $c_{4}, c_{6}$ et $\Delta$ d'une courbe elliptique', Acta Arith. 54 (1989), 75-80.

[5] S. Siksek, 'Infinite descent on elliptic curves', Rocky Mountain J. Math. 25 (1995), 1501-1538.

[6] J.H. Silverman, 'Lower bounds for height functions', Duke Math. J. 51 (1984), 395-403.

[7] J.H. Silverman, The arithmetic of elliptic curves, Graduate Texts in Mathematics 106 (Springer-Verlag, New York, 1986).

[8] J.H. Silverman, Rational points on certain families of curves of genus at least 2, Proc. London Math. Soc. 55 (1987), 465-481.

[9] D. Simon, 'Programme de calcul du rang des courbes elliptiques dans les corps de nombres, disponible à l'adresse', http://wrw .math.unicaen.fr/ ${ }^{-}$simon/.

[10] J. Tate, 'Algorithm for determining the type of a singular fiber in an elliptic pencil, dans Modular Functions of One Variable IV', Lecture Notes in Math. 476 (1975), 33-52.

App. 231

9 rue de Sèvres

92100 Boulogne

France

e-mail: elie.cali@wanadoo.fr
Université Pierre et Marie Curie - Paris 6

Institut de Mathématiques

UMR 7586 du CNRS

175 rue du Chevaleret

75013 Paris

France

e-mail: kraus@math.jussieu.fr 